\title{
Hydroquinones with Conformationally Constrained Substituents: Synthesis, Characterization, and Evaluation as Calcium-ATPase Inhibitors
}

\author{
S. Paula, C. Elam, M. Woeste, J. Abell, and R. J. Kempton
}

\begin{abstract}
Derivatives of the compound 2,5-di-tert-butylhydroquinone (BHQ) are inhibitors of the sarco/endoplasmic reticulum calcium ATPase (SERCA). Previous work identified BHQ analogs with two alkyl substituents composed of four to six carbon atoms as the most potent representatives from this inhibitor class. In this study, we explored the effects of introducing two cycloalkyl substituents on the hydroquinone scaffold. Since these substituents have limited conformational flexibility, the entropy penalty upon binding of these compounds to SERCA was expected to be smaller than for non-cyclic BHQ analogs, potentially conveying higher inhibitory potency. We synthesized a congeneric series of four 2,5-disubstituted BHQ analogs and determined their inhibitory potencies against SERCA in bioassays. Potencies were found in the low micromolar and submicromolar concentration ranges, making the new compounds some of the most potent hydroquinone-based inhibitors to date. Computational docking facilitated a detailed analysis of enzyme/inhibitor interactions at the molecular level and showed that the entropic effect was noticeable but too small to increase potency in a substantial manner.
\end{abstract}

Index Terms-Calcium homeostasis, enzyme inhibition, P-type ATPase, medicinal chemistry.

\section{INTRODUCTION}

The natural product thapsigargin (TG), extracted from the Mediterranean plant Thapsia garganica, is a highly potent and specific inhibitor of the enzyme sarco/endoplasmic reticulum calcium ATPase (SERCA). For several decades, TG has been an indispensable tool for the study of SERCA's physiological functions. More recently, it has also been used for the generation of prodrugs for prostate cancer chemotherapy [1]-[4]. Even though TG remains the most potent SERCA inhibitor to date, its restricted availability has limited its use. Extraction yields from its natural source are low [5] and TG's structural complexity makes total synthesis - a feat accomplished in about 40 steps - an unfeasible route to larger quantities of this compound [6], [7]. As a result, searches for alternative SERCA inhibitors that are readily available are currently ongoing.

A sizeable number of structurally diverse compounds are

Manuscript received March 26, 2013; revised May 28, 2013. This work was supported in part by grants from the National Institute of General Medical Sciences (8P20GM103436-12 and 1R15GM084431-01).

The authors are with the Department of Chemistry at Northern Kentucky University, Highland Heights, KY 41099, U.S.A. (e-mail: paulas1@nku.edu). capable of inhibiting SERCA, albeit with potencies lower than that of TG. Examples include, but are not limited to, the fungal metabolite cyclopiazonic acid, bisphenols, curcumins, and hydroquinones [8], [9]. The most prominent representative from the latter class is the compound 2,5-di-tert-butylhydroquinone (BHQ) [10]-[13]. Due to their relatively small size, BHQ derivatives can be synthesized in a few steps from inexpensive starting materials, which constitutes a considerable advantage over TG-based inhibitors. Structure-activity relationships are available for sizeable collections of hydroquinones obtained from virtual screens of large compound collections or from organic synthesis [13]-[15]. Among the hydroquinones tested in enzyme inhibition assays, the highest potency inhibitors with submicromolar potencies are 2,5-disubstituted compounds that carry two alkyl residues with four to six carbon atoms each.

In an effort to design novel hydroquinone-based SERCA inhibitors with improved potencies, we explored the concept of limiting the conformational flexibility of inhibitors by introducing cyclic substituents. The underlying rationale was that conformationally restrained, rigid substituents would experience a lower loss in conformational entropy upon binding to SERCA, thereby giving rise to a more favorable binding energy and thus a higher inhibitory potency. This effect is based on the fact that in solution, a flexible ligand has a higher entropy than an analogous rigid ligand of similar size. Upon binding, both ligands are confined to the inside of the protein's binding site, which restricts their dynamic motions and occupancy of multiple conformational states, resulting in a overall more ordered state of lower entropy. However, the relative loss of conformational entropy upon binding is lower for the rigid ligand. This concept of optimizing ligand affinity by constraining conformational entropy is well-known and has been evaluated in various systems with ligands such as peptides or carbohydrates [16]-[18].

Using Friedel-Crafts alkylation methodology [19] with commercially available hydroquinone and cyclic alcohols, we synthesized four BHQ analogs with four- to seven-membered rings and determined their inhibitory potencies against SERCA activity in bioassays. An analysis of enzyme/inhibitor interactions was performed with the aid of molecular docking. The latter is a commonly used computational method that predicts the most favorable pose of a given ligand in a receptor binding site. By identifying the intermolecular interactions that contribute to binding of a ligand, docking provides an account of ligand binding at the molecular level. 


\section{MATERIALS AND MethodS}

\section{A. Organic Synthesis of Hydroquinones}

Chemicals. All reagents were purchased from Sigma-Aldrich (St. Louis, MO) or Fisher Scientific (Pittsburgh, PA) and were used without further purification.

Synthesis. The syntheses of the disubstituted analogs of hydroquinone were carried out under an argon atmosphere under one of the following three conditions: glacial acetic acid/concentrated sulfuric acid $(5: 1)$, initially at $0{ }^{\circ} \mathrm{C}$, then warming to room temperature and stirring for $24 \mathrm{~h}$ (Method A); $75 \%$ aqueous sulfuric acid at $0^{\circ} \mathrm{C}$ for $1 \mathrm{~h}$ (Method B); glacial acetic acid at $50^{\circ} \mathrm{C}$ for $4 \mathrm{~h}$ (Method C). All reactions were quenched in ice and the reaction products partitioned between water and ethyl acetate. The organic layer was washed twice with water, dried $\left(\mathrm{Na}_{2} \mathrm{SO}_{4}\right)$, and concentrated by rotary evaporation. Purification was accomplished by preparative thin layer chromatography (TLC; silica gel, 10\% ethyl acetate in hexane). Proton (500 $\mathrm{MHz}$ ) and carbon (125.7 MHz) NMR spectra were obtained on a JEOL Eclipse 500 spectrometer (Peabody, MA). Chemical shifts are reported in $\delta$ values relative to TMS in $\mathrm{CDCl}_{3}$. Melting points were recorded on a Thomas Hoover instrument and are uncorrected. High resolution mass spectra (HRMS) were obtained from the mass spectrometry facility at the University of Illinois at Urbana.

\section{1) 2,5-bis-(1'-methylcyclobutyl)-1,4-hydroquinone}

Method C. HRMS: calculated for $\mathrm{C}_{16} \mathrm{H}_{22} \mathrm{O}_{2}$ : 246.16198; found: $246.16258 .{ }^{1} \mathrm{H}-\mathrm{NMR}: \delta 1.47(\mathrm{~s}, 6 \mathrm{H}), 1.85(\mathrm{~m}, 2 \mathrm{H})$, $2.05(\mathrm{~m}, 4 \mathrm{H}), 2.17(\mathrm{~m}, 2 \mathrm{H}), 2.42(\mathrm{~m}, 4 \mathrm{H}), 4.14(\mathrm{~s}, 2 \mathrm{H}), 6.38$ (s, 2H). ${ }^{13} \mathrm{C}-\mathrm{NMR}: \delta$ 16.38, 27.40, 33.98, 41.02, 114.01, 135.45, 146.56. mp: $176-178^{\circ} \mathrm{C}$. Yield: $15 \%$.

\section{2) 2,5-bis-(1'-methylcyclopentyl)-1,4-hydroquinone}

Method B. HRMS: calculated for $\mathrm{C}_{18} \mathrm{H}_{26} \mathrm{O}_{2}$ : 274.1933; found: $274.1932 .{ }^{1} \mathrm{H}-\mathrm{NMR}: \delta 1.25(\mathrm{~s}, 6 \mathrm{H}), 1.73(\mathrm{~m}, 8 \mathrm{H}), 1.88$ $(\mathrm{m}, 8 \mathrm{H}), 4.50(\mathrm{bs}, 2 \mathrm{H}), 6.61(\mathrm{~s}, 2 \mathrm{H}) .{ }^{13} \mathrm{C}-\mathrm{NMR}: \delta 23.66$, 26.58, 38.81, 45.29, 115.69, 134.72, 147.11. mp: $179-182^{\circ} \mathrm{C}$. Yield: $42 \%$.

\section{3) 2,5-bis-(I'-methylcyclohexyl)-1,4-hydroquinone}

Method B. ${ }^{1} \mathrm{H}-\mathrm{NMR}: \delta 1.25(\mathrm{~s}, 6 \mathrm{H}), 1.32-1.63(\mathrm{~m}, 16 \mathrm{H})$, $1.83(\mathrm{~m}, 4 \mathrm{H}), 4.41(\mathrm{~s}, 2 \mathrm{H}), 6.47(\mathrm{~s}, 2 \mathrm{H}) .{ }^{13} \mathrm{C}-\mathrm{NMR}: \delta 22.84$, $25.45,26.62,36.89,37.40,116.88,133.61,147.58 . \mathrm{mp}:$ $169-172^{\circ} \mathrm{C}$. (literature value: $168-174^{\circ} \mathrm{C}$ [20] ). Yield: $39 \%$.

\section{4) 2,5-bis-(1'-methylcycloheptyl)-1,4-hydroquinone}

Method A. HRMS: calculated for $\mathrm{C}_{22} \mathrm{H}_{34} \mathrm{O}_{2}$ : 330.25588; found: $330.25676 .{ }^{1} \mathrm{H}-\mathrm{NMR}: \delta 1.28(\mathrm{~s}, 6 \mathrm{H}), 1.54(\mathrm{~m}, 16 \mathrm{H})$, $1.78(\mathrm{~m}, 4 \mathrm{H}), 2.12(\mathrm{~m}, 4 \mathrm{H}), 4.33(\mathrm{~s}, 2 \mathrm{H}), 6.53(\mathrm{~s}, 2 \mathrm{H})$. ${ }^{13} \mathrm{C}-\mathrm{NMR}: \delta$ 23.87, 28.39, 30.24, 39.61, 41.04, 116.73, 134.20, 147.41. mp: $145-146^{\circ} \mathrm{C}$. Yield: $36 \%$.

\section{B. Measurement of Inhibitory Potencies}

Inhibitory potencies of the four synthesized BHQ analogs were determined spectroscopically in a coupled assay using SERCA prepared as rabbit microsomes as described previously [14], [15]. Rates of SERCA-catalyzed ATP hydrolysis coupled to NADH oxidation via the action of pyruvate kinase and lactate dehydrogenase were measured at different inhibitor concentrations. A three-parameter logistic fit of activity versus inhibitor concentration data then yielded the inhibitory potency expressed as the $\mathrm{IC}_{50}$ value, the inhibitor concentration reducing enzyme activity by $50 \%$ [21]. The four compounds were also tested in a second, independent assay that directly monitored the SERCA-catalyzed production of inorganic phosphate using the dye Malachite Green [22]. This type of assay was conducted at only two or three inhibitor concentrations and served the purpose of ruling out the possibility of an inhibitor interfering with the auxiliary enzymes in the coupled assay, rather than inhibiting SERCA directly.

\section{Computational Ligand Docking}

Docking was performed with the program GOLD (version 5.1; Cambridge Crystallographic Data Centre, UK) [23, 24] and the X-ray crystal structure of the SERCA/BHQ complex (Protein Data Bank entry 2AGV) [25]. The protein structure was prepared for docking by removing all non-protein entries, adding hydrogen atoms, and optimizing the position of the latter by molecular mechanics as described previously [13, 26]. The scoring function used for docking was ChemScore $[27,28]$ and the genetic algorithm of GOLD was executed at the default settings, performing 20 independent and identical repeats for each compound. The docking sphere had a radius of $15 \AA$ centered at the location of the $\mathrm{C}-1$ phenyl carbon of the deleted BHQ in the SERCA/BHQ complex. The structures of inhibitors were modeled in MOE (version 2012.10; Chemical Computing Group, Montreal, Canada) and energy-minimized with the MMFF94s force field. Results were analyzed with the GOLD tool Hermes and enzyme/inhibitor interactions were visualized in schematic diagrams created in MOE.

\section{RESULTS AND DISCUSSION}

\section{A. Synthesis of 2,5-Disubstituted Hydroquinones}

As illustrated in Fig. 1, a series of four 2,5-disubstituted hydroquinones with cyclic substituents was synthesized by Friedel-Crafts alkylation of hydroquinone with commercially available cyclic tertiary alcohols. Since previous studies had shown that the most active hydroquinone-based SERCA inhibitors with non-cyclic residues carried between four and six carbon atoms in their side chains [10], [13], we varied the ring size of the newly synthesized compounds from four to seven carbon atoms to be within this size range.

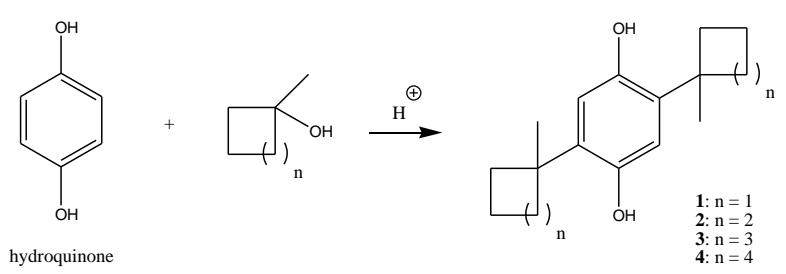

Fig. 1. Synthesis of disubstituted hydroquinones with cyclic substituents.

Disubstituted hydroquinones 1-4 were prepared in low yields by small scale (100-200 $\mathrm{mg}$ ) reaction of hydroquinone with slightly more than two equivalents of the appropriate cycloalkanol under acidic conditions. No efforts were made to optimize reaction yields. In the case of the four-membered analog $\mathbf{1}$, the cycloalkanol was not commercially available. Consequently, hydroquinone was reacted with 
methylenecyclobutane in glacial acetic acid at $50^{\circ} \mathrm{C}$ for $4 \mathrm{~h}$ to obtain 1. All analogs were characterized by ${ }^{1} \mathrm{H}-$ and ${ }^{13} \mathrm{C}-\mathrm{NMR}$. In addition, HRMS was performed for all compounds, except for analog 3 (six-membered substituent), which is a known compound [20]. Several attempts were made to prepare the bis-(1'-methylcyclooctyl)-1,4hydroquinone analog, and in one such attempt, enough material was isolated for HRMS (calculated for $\mathrm{C}_{24} \mathrm{H}_{38} \mathrm{O}_{2}$ : 358.28718; found: 358.28696). The ${ }^{1} \mathrm{H}-$ and ${ }^{13} \mathrm{C}-\mathrm{NMR}$ spectra of this material were consistent with the desired bis-cyclooctyl product, but to date not enough sample of this compound has been obtained for biological testing. Also appearing as a minor component of this reaction was a product whose ${ }^{1} \mathrm{H}-\mathrm{NMR}$ exhibits two signals in the aromatic region of the spectrum at $\delta 6.04$ and 6.66 with relative integration areas of $1: 2$, suggesting that it is the mono-alkylated 2-(1'-methylcyclooctyl)-1,4-hydroquinone. Efforts to prepare the cyclooctyl analog in sufficient quantity for testing, along with the preparation of other analogs, are currently in progress.

\section{B. Inhibitory Potencies and Structure-Activity Trends}

Fig. 2 depicts a representative inhibition curve obtained for 2, the most potent compound in the series. All four hydroquinones were able to inhibit SERCA with excellent potencies, which were measured in the low micromolar or high nanomolar concentration range (Table I). 2, the hydroquinone with 5-membered rings, was the most potent one, followed by the larger $\mathbf{3}$ and the smaller $\mathbf{1}$. The least potent hydroquinone derivative was $\mathbf{4}$, which carried the largest substituents. The observed trend suggested an optimum number of six carbon atoms total (five ring plus one methyl carbon atom) in the substituent, a size which is most likely dictated by the size of the binding pocket. It should be noted that $\mathbf{4}$ is about as potent as the most active non-cyclic hydroquinone-based SERCA inhibitor known to date, 2,5-diamylhydroquinone $\left(\mathrm{IC}_{50}: 0.34 \mu \mathrm{M}\right)$ [10], [29].

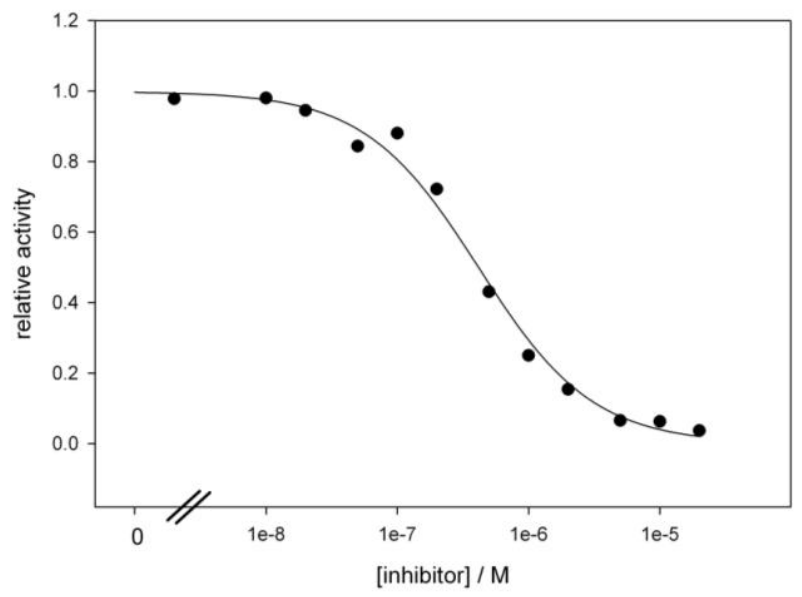

Fig. 2. Representative SERCA inhibition assay with 2. Symbols indicate measured ATP hydrolysis rates and the solid line was obtained from a three-parameter logistic fit to the data.

\section{Analysis of Inhibitor Binding by Computational Docking}

After the structures of 1-4 had been modeled, they were docked into the X-ray crystal structure of SERCA in a conformation representative of the enzyme in the $E_{2}$ state. The latter had been identified as the relevant conformation for binding inhibitors such as BHQ [25]. A previously developed and validated docking protocol for hydroquinone/SERCA with the program GOLD was employed [26]. For comparison purposes, the highly active, non-cyclic 2,5-diamylhydroquinone was included in the docking runs as well. All compounds gave excellent consensus orientations, implying that docking runs identical conditions generated the same ligand pose. Moreover, all hydroquinones were predicted to bind in the same orientation to the enzyme (Fig. 3 A), thus sharing a common binding mode.

TABLE I: INHIBITORY POTENCIES AND DOCKING-PREDICTED BINDING ENERGIES OF SYNTHESIZED SERCA INHIBITORS

\begin{tabular}{cccc}
\hline \hline \multirow{2}{*}{ compound } & $\mathrm{IC}_{50}(\mu \mathrm{M})$ & \# of trials & ChemScore $/ \mathrm{kJ} \mathrm{mol}^{-1}$ \\
\hline $\mathbf{1}$ & $2.5 \pm 0.3$ & 4 & 33.3 \\
$\mathbf{2}$ & $0.52 \pm 0.39$ & 9 & 36.2 \\
$\mathbf{3}$ & $2.2 \pm 1.3$ & 8 & 33.9 \\
$\mathbf{4}$ & $9.4 \pm 3.1$ & 4 & 31.3 \\
\hline \hline
\end{tabular}
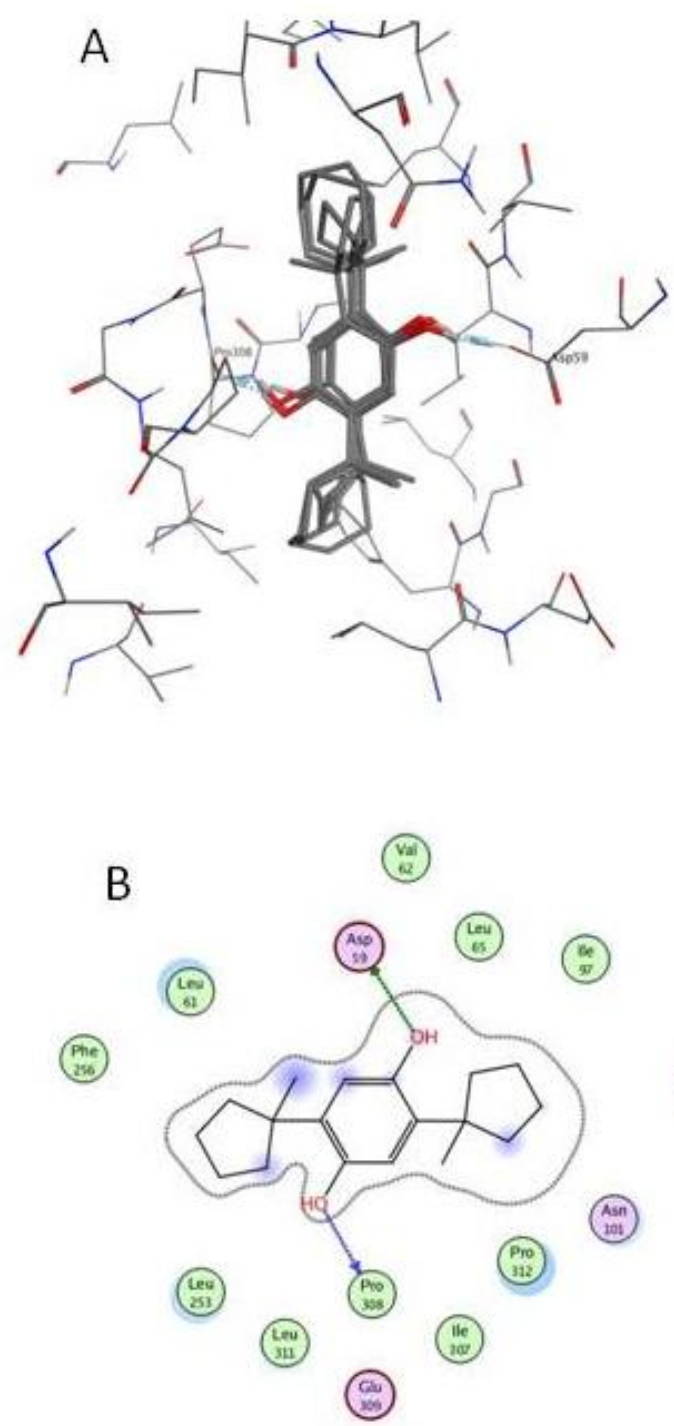

Fig. 3. Intermolecular interactions between SERCA and inhibitors as predicted by docking. A: Superimposition of the top-scoring poses for compounds 1-4. B: Schematic representation of hydrogen bonds (broken lines) and hydrophobic residues (green) in the binding pocket for $\mathbf{2}$. Blue shadows indicate solvent exposure. 
Similar to BHQ binding to SERCA, the two hydroxyl groups of the inhibitors were engaged in hydrogen bonds with Asp59 and Pro308. The non-polar substituents were accommodated in areas of the binding pocket lined by hydrophobic residues (Fig. 3 B). This binding pose suggests that inhibition by these compounds is achieved by locking SERCA in its $E_{2}$ conformation, thereby preventing it from undergoing additional catalytic cycles. The latter effect is believed to be the result of cross-linking Asp59 with Pro308, which prevents the relative movement of two of SERCA's transmembrane helices. In addition, inhibitor binding causes Glu309 to assume a position that blocks a crucial calcium channel inside the enzyme.

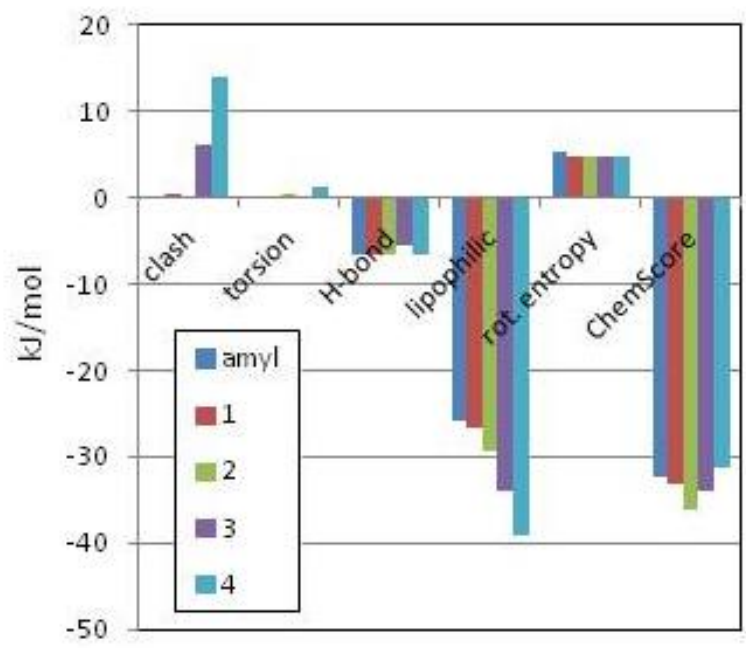

Fig. 4. Intermolecular interactions between SERCA and inhibitors as predicted by docking. Breakdown of individual energy contributions to the total binding energy as predicted by ChemScore.

Next, the ChemScore values obtained for the top-scoring solution for each compound were compared to the experimentally determined potencies to identify potential trends (Table I). ChemScore was designed to correlate with binding energies [27], [28]. The structural similarity of the four hydroquinones strongly suggests that they employ the same inhibition mechanism [25], leaving variations in binding energies as the sole source of differing inhibitory potencies. As anticipated, the most active $\mathbf{2}$ also had the highest ChemScore value whereas the least active $\mathbf{4}$ had the lowest. Fig. 4 provides a breakdown of the various contributions to the ChemScore term, identifying hydrophobic interactions as the dominant binding force, followed by hydrogen bonding. While the large and favorable hydrophobic term increased with increasing size of the substituents, it was opposed by clash penalties for the two larger inhibitors $\mathbf{3}$ and $\mathbf{4}$, which resulted in a somewhat diminished overall binding energy.

Fig. 4 shows that the docking-predicted contribution stemming from a loss of conformational entropy did not vary greatly among cyclic inhibitors. In comparison to inhibitor $\mathbf{1}$, the isosteric non-cyclic diamylhydroquinone experienced a slightly larger entropy penalty, exactly as anticipated (5.3 versus $4.8 \mathrm{~kJ} / \mathrm{mol}$ ). However, the slightly larger entropy penalty of diamylhydroquinone was overcompensated by the favorable hydrophobic term, which was larger for this less compact, non-cyclic molecule. As a general pattern, the reduction of the unfavorable entropy term seen for cyclic inhibitors was rather small and did not appear to have a major impact on the overall behavior of the compounds.

\section{CONCLUSIONS}

In this study, we developed straightforward synthetic routes that provide convenient access to 2,5-disubstituted hydroquinones with cyclic substituents. Using bioassays, we evaluated the compounds' potential as SERCA inhibitors. Whereas the measured potencies were excellent and rivaled those of the most potent known hydroquinone-based inhibitors, they did not exceed them. Docking showed that the introduction of cyclic substituents slightly reduced the entropy penalty for binding of conformationally constrained inhibitors to the enzyme relative to non-cyclic inhibitors of similar size. Somewhat disappointingly though, computational docking results showed that the magnitude of the entropic effect was insufficient to result in a significant gain in potency, a prediction that was consistent with the measured inhibitory potencies.

\section{REFERENCES}

[1] J. Isaacs, "New strategies of the medical treatment of prostate cancer," BJU International, vol. 96, 2005, pp. 35-40.

[2] M. Treiman, C. Caspersen, and S. B. Christensen, "A tool coming of age: thapsigargin as an inhibitor of sarco-endoplasmic reticulum $\mathrm{Ca}^{2+}$-ATPases," Trends Pharmacol Sci, vol. 19, 1998, pp. 131-135.

[3] U. Rasmussen, S. Broogger Christensen, and F. Sandberg, "Thapsigargine and thapsigargicine, two new histamine liberators from Thapsia garganica L," Acta Pharm Suec, vol. 15, 1978, pp. 133-140.

[4] S. R. Denmeade and J. T. Isaacs, "The SERCA pump as a therapeutic target: making a 'smart bomb' for prostate cancer," Cancer Biol Ther, vol. 4, 2005, pp. 14-22.

[5] S. B. Christensen, U. Rasmussen, and C. Christophersen, "Thapsigargin, constitution of a sesquiterpene lactone histamine liberator from thapsia garganica," Tetrahedron Letters, vol. 21, 1980, pp. 3829-3830.

[6] M. Ball, S. P. Andrews, F. Wierschem, E. Cleator, M. D. Smith, and S V. Ley, "Total synthesis of thapsigargin, a potent SERCA pump inhibitor," Organic Letters, vol. 9, 2007, pp. 663-666.

[7] S. P. Andrews, M. M. Tait, M. Ball, and S. V. Ley, "Design and total synthesis of unnatural analogues of the sub-nanomolar SERCA inhibitor thapsigargin," Org Biomol Chem, vol. 5, 2007, pp. 1427-1436.

[8] S. Paula, "Progress in the Discovery of SERCA Inhibitors," in G. P. Colombo, S. Ricci (Ed.), Medicinal Chemistry Research Progress, Nova Science Publishers, Inc., 2008.

[9] F. Michelangeli and J. M. East, "A diversity of SERCA Ca ${ }^{2+}$ pump inhibitors," Biochemical Society transactions, vol. 39, 2011, pp. 789-797.

[10] Y. M. Khan, M. Wictome, J. M. East, and A. G. Lee, "Interactions of dihydroxybenzenes with the $\mathrm{Ca}^{2+}$-ATPase: separate binding sites for dihydroxybenzenes and sesquiterpene lactones," Biochemistry, vol. 34, 1995, pp. 14385-14393.

[11] G. A. Moore, D. J. McConkey, G. E. Kass, P. J. O'Brien, and S. Orrenius, "2,5-Di(tert-butyl)-1,4-benzohydroquinone - a novel inhibitor of liver microsomal $\mathrm{Ca}^{2+}$ sequestration," FEBS letters, vol. 224, 1987, pp. 331-336.

[12] M. Wictome, M. Holub, J. M. East, and A. G. Lee, "The importance of the hydroxyl moieties for inhibition of the $\mathrm{Ca}^{2+}$-ATPase by trilobolide and 2,5-di(tert-butyl)-1,4-benzohydroquinone," Biochemical and biophysical research communications, vol. 199, 1994, pp. 916-921.

[13] M. Lape, C. Elam, M. Versluis, R. Kempton, and S. Paula, "Molecular determinants of sarco/endoplasmic reticulum calcium ATPase inhibition by hydroquinone-based compounds," Proteins, vol. 70, 2008, pp. 639-649

[14] J. Deye, C. Elam, M. Lape, R. Ratliff, K. Evans, and S. Paula, "Structure-based virtual screening for novel inhibitors of the sarco/endoplasmic reticulum calcium ATPase and their experimental evaluation," Bioorg Med Chem, vol. 17, 2009, pp. 1353-1360. 
[15] C. Elam, M. Lape, J. Deye, J. Zultowsky, D. T. Stanton, and S. Paula, "Discovery of novel SERCA inhibitors by virtual screening of a large compound library," Eur J Med Chem, vol. 46, 2011, pp. 1512-1523.

[16] D. G. Udugamasooriya and M. R. Spaller, "Conformational constraint in protein ligand design and the inconsistency of binding entropy," Biopolymers, vol. 89, 2008, pp. 653-667.

[17] A. R. Khan, J. C. Parrish, M. E. Fraser, W. W. Smith, P. A. Bartlett, and M. N. James, "Lowering the entropic barrier for binding conformationally flexible inhibitors to enzymes," Biochemistry, vol. 37 1998, pp. 16839-16845.

[18] N. Navarre, N. Amiot, A. van Oijen, A. Imberty, A. Poveda, J. Jimenez-Barbero, A. Cooper, M. Jutley, and G.-J. Boons, "Synthesis and conformational analysis of a conformationally constrained trisaccharide, and complexation properties with concanavalin A," Chem. Eur. J., vol. 5, 1999, pp. 2281-2294.

[19] G. A. Olah, Friedel-Crafts Chemistry, New York: Wiley, 1973.

[20] N. C. Deno and H. Chafetz, "Alkylation of hydroquinone and its derivatives," J. Org. Chem., vol. 19, 1954, pp. 2019-2022.

[21] Y. Cheng and W. H. Prusoff, "Relationship between the inhibition constant (K1) and the concentration of inhibitor which causes 50 per cent inhibition (I50) of an enzymatic reaction," Biochem Pharmacol vol. 22. 1973. pp. 3099-3108.

[22] P. V. Veldhoven and G. Mannaerts, "Inorganic and organic phosphate measurements in the nanomolar range," Analytical Biochemistry, vol 161, 1987, pp. 45-48.

[23] G. Jones, P. Willett, and R. C. Glen, "Molecular recognition of receptor sites using a genetic algorithm with a description of desolvation," $J \mathrm{Mol}$ Biol, vol. 245, 1995, pp. 43-53.

[24] G. Jones, P. Willett, R. C. Glen, A. R. Leach, and R. Taylor, "Development and validation of a genetic algorithm for flexible docking," J Mol Biol, vol. 267, 1997, pp. 727-748.

[25] K. Obara, N. Miyashita, C. Xu, I. Toyoshima, Y. Sugita, G. Inesi, and C. Toyoshima, "Structural role of countertransport revealed in $\mathrm{Ca}^{2+}$ pump crystal structure in the absence of $\mathrm{Ca}^{2+}$," in Proc. the National Academy of Sciences of the United States of America, vol. 102, 2005, pp. 14489-14496.

[26] M. Lape, C. Elam, and S. Paula, "Comparison of current docking tools for the simulation of inhibitor binding by the transmembrane domain of the sarco/endoplasmic reticulum calcium ATPase," Biophys Chem, vol. 150, 2010, pp. 88-97.

[27] C. A. Baxter, C. W. Murray, D. E. Clark, D. R. Westhead, and M. D. Eldridge, "Flexible docking using Tabu search and an empirical estimate of binding affinity," Proteins, vol. 33, 1998, pp. 367-382.

[28] M. D. Eldridge, C. W. Murray, T. R. Auton, G. V. Paolini, and R. P. Mee, "Empirical scoring functions: I. The development of a fast empirical scoring function to estimate the binding affinity of ligands in receptor complexes," J Comput Aided Mol Des, vol. 11, 1997, pp. 425-445.

[29] S. Paula, J. Abell, J. Deye, C. Elam, M. Lape, J. Purnell, R. Ratliff, K. Sebastian, J. Zultowsky, and R. J. Kempton, "Design, synthesis, and biological evaluation of hydroquinone derivatives as novel inhibitors of the sarco/endoplasmic reticulum calcium ATPase," Bioorg Med Chem, vol. 17, 2009, pp. 6613-6619.

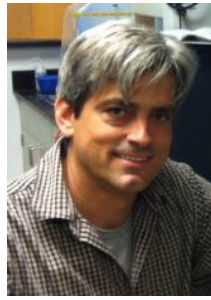

Stefan Paula is a native of Germany, who obtained his diploma in chemistry at the University of Kaiserslautern, Germany, in 1992. He then spent one year as a Fulbright Fellow in the United States at the University of California at Davis. He received his Ph.D. in chemistry in 1998 from the University of California at Santa Cruz. After postdoctoral training at the Max Planck Institute for Biochemistry, Germany, and the University of Cincinnati College of Medicine in the United States, he became a faculty member at Northern Kentucky University (Highland Heights, Kentucky) in 2004, where he is currently an associate professor of chemistry.

His research interests are in the area of computer-assisted drug design. His group uses a combination of experimental and computational methodologies to gain an understanding of how small bioactive molecules interact with their protein receptors. The ultimate goal is the design of novel molecules with improved bioactivities. Several enzyme/inhibitor systems are currently under study, including inhibitors of the sarco/endoplasmic reticulum calcium ATPase, the sodium/potassium ATPase, xanthine oxidase, and aromatase. The preferred approach entails a computational analysis of the interactions between a compound class and the receptor, followed by virtual screening of large compound libraries with predictive models and experimental testing of selected molecules in bioassays. To gain access to specific molecules, his research group collaborates with several synthetic organic chemists.

Dr. Paula is a member of the American Chemical Society and the Council on Undergraduate Research

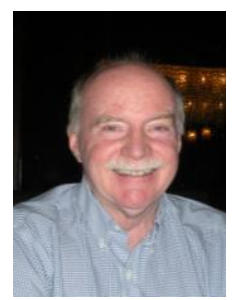

Robert Kempton was born in Brooklyn, New York He received a B.S. in chemistry from St. Peter's College, New Jersey, in 1966, followed by a M.S. in organic chemistry from Auburn University, Alabama, in 1969. He obtained a Ph.D. in organic chemistry from Fordham University, New York, in 1975.

He worked as a research chemist at CIBA Pharmaceutical (now Norvartis) in Summit, New Jersey, from 1969-1971. In 1976, he joined the faculty of Northern Kentucky University (Highland Heights, Kentucky) and currently holds the title of Regent Professor of Chemistry Emeritus. He has held postdoctoral positions in the chemistry departments of the University of Illinois at Urbana, Princeton University, and the University of Cincinnati. His research interests have been focused on the synthesis of analogs of the anti-cancer drug methotrexate and most recently on the synthesis of analogs of 2,5-di-tert-butylhydroquinone.

Dr. Kempton is a member of the American Chemical Society and the Council on Undergraduate Research. 